\title{
CUT POINTS IN ČECH-STONE REMAINDERS
}

\author{
ALAN DOW AND KLAAS PIETER HART
}

(Communicated by Franklin D. Tall)

\begin{abstract}
We investigate cut points of subcontinua of $\beta \mathbb{R} \backslash \mathbb{R}$. We find, under $\mathrm{CH}$, the topologically smallest type of subset of $\mathbb{R}$ that can support such a cut point. On the other hand we answer Question 66 of Hart and van Mill's Open problems on $\beta \omega$ [Open Problems in Topology (J. van Mill and G. M. Reed, eds.), North-Holland, Amsterdam, 1990, pp. 97-125] by showing that it is consistent that all cut points are trivial (in a sense to be made precise in the paper).
\end{abstract}

\section{INTRODUCTION}

In this paper we study some types of points of $\mathbb{H}^{*}$, where $\mathbb{H}$ is the half line $[0, \infty)$. It is well known that $\mathbb{H}^{*}$ is an indecomposable continuum. As such it does not have any cut points, but it does have sub cut points, i.e., points that are cut points of some subcontinuum.

To find sub cut points one only has to look in $\omega^{*}$ : for each $n$ let $\mathbb{I}_{n}$ be the interval $[n-1 / 4, n+1 / 4]$ and for $u \in \omega^{*}$ put $\mathbb{I}_{u}=\bigcap_{U \in u} \operatorname{cl}_{n \in U} \mathbb{I}_{n}$. It is readily verified that $\mathbb{I}_{u}$ is a continuum and that $u$ is a cut point of it. This argument shows that every point that is in the closure of a closed and discrete subset of $\mathbb{H}$ (a so-called near point) is in fact a sub cut point.

On the other hand, $\mathbb{H}^{*}$ also has points that are not sub cut points; such points were found by van Douwen in [2] and van Mill and Mills in [7]. This gives a clear cut reason why the space $\mathbb{H}^{*}$ is not homogeneous: there are points with visibly different topological behaviour.

It can also be shown that if $x$ is a sub cut point of $\mathbb{H}^{*}$ then there are a discrete sequence $\left\langle\mathbb{I}_{n}: n \in \omega\right\rangle$ of closed intervals in $\mathbb{H}$ and a $u \in \omega^{*}$ such that $x$ is a cut point of $\mathbb{I}_{u}$.

These results prompted further investigation of the structure of the continua $\mathbb{I}_{u}$. One question - mentioned as Question 66 (or Problem 265) in Hart and van Mill [5]-was whether every cut point of $\mathbb{I}_{u}$ is of the form $u$ - $\lim x_{n}$, where $\left\langle x_{n}: n \in \omega\right\rangle$ is a sequence such that $x_{n} \in \mathbb{I}_{n}$ for all $n$; for clearly every

Received by the editors November 20, 1992 and, in revised form, March 30, 1993.

1991 Mathematics Subject Classification. Primary 54F15; Secondary 54F50, 54A35, 03 E50.

Key words and phrases. Čech-Stone compactification, continuum, cut point, Laver forcing.

The results of this note were obtained while the second author was visiting York University. Thanks are due to all who made this visit possible. 
such point is a cut point of $\mathbb{I}_{u}$. Let us call such points trivial cut points. We shall abbreviate $u$-lim $x_{n}$ by $x_{u}$ (the $u$-th term of the sequence).

Nontrivial cut points were constructed by Baldwin and Smith in [1] and by Zhu in [9] using Martin's Axiom for Countable posets and $\mathrm{CH}$, respectively.

The main result of our paper shows that in Laver's model for the Borel Conjecture (Laver [6]) no $\mathbb{I}_{u}$ has a nontrivial cut point. This confirms a conjecture mentioned in the paper of Hart and van Mill.

The paper is organized as follows: In Section 1 we summarize some known facts about cut points of $\mathbb{I}_{u}$, needed in Section 2. We also prove, under $\mathrm{CH}$, a result that shows that every $\mathbb{I}_{u}$ may have a nontrivial cut point and that also identifies, in some sense, the topologically smallest type of set that can support a nontrivial cut point (Proposition 1.4 and Theorem 1.5 will give a meaning to the phrase 'topologically smallest'). In Section 2 we prove our main result and interpret it in terms of $\mathbb{H}^{*}$.

The survey in [4] will provide proofs for statements not proven here. In that paper a sub cut point is called a weak cut point. The term sub cut point seems more appropriate.

\section{VARIOUS KINDS OF CUT POINTS}

For convenience we consider the space $\mathbb{M}=\omega \times \mathbb{I}$, where $\mathbb{I}=[0,1]$. We write $\mathbb{I}_{n}=\{n\} \times \mathbb{I}$ and we interpret $\mathbb{I}_{u}$ accordingly.

As we shall have no reason to take a union of a sequence of intervals in $\mathbb{I}$ itself we shall relieve our notational burden somewhat by agreeing that $\bigcup_{n}\left[a_{n}, b_{n}\right]$ means $\bigcup_{n}\{n\} \times\left[a_{n}, b_{n}\right]$, whenever $\left\langle\left[a_{n}, b_{n}\right]: n \in \omega\right\rangle$ is a sequence of intervals in $\mathbb{I}$.

We begin by giving the most convenient-for us-characterizations of cut points in the continua $\mathbb{I}_{u}$. Let $x \in \mathbb{I}_{u}$. We define two subsets $A_{x}$ and $B_{x}$ of $\mathbb{I}^{\omega}$ as follows:

$$
A_{x}=\left\{a \in \mathbb{I}^{\omega}: x \in \operatorname{cl} \bigcup_{n \in \omega}[a(n), 1]\right\}
$$

and

$$
B_{x}=\left\{b \in \mathbb{I}^{\omega}: x \in \mathrm{cl} \bigcup_{n \in \omega}[0, b(n)]\right\},
$$

where the closures are taken in $\beta \mathrm{M}$ of course.

Now if $x$ is of the form $x_{u}$ for some sequence $\left\langle x_{n}: n \in \omega\right\rangle$ in $\mathbb{I}^{\omega}$ then $A_{x} \cap B_{x}$ consists exactly of those sequences $\left\langle y_{n}: n \in \omega\right\rangle$ for which there is $U \in u$ such that $x(n)=y(n)$ for all $n \in U$. Otherwise the intersection of $A_{x}$ and $B_{x}$ is empty.

Thus the trivial cut points of $\mathbb{I}_{u}$ are characterized by the fact that $A_{x} \cap B_{x} \neq$ $\varnothing$. The following proposition characterizes the nontrivial cut points in terms of $A_{x}$ and $B_{x}$. If $U$ is an open subset of $\mathbb{M}$ then $\operatorname{Ex} U$ denotes the largest open subset of $\beta \mathrm{M}$ whose intersection with $\mathbb{M}$ is $U$. Let us call a sequence $\left\langle r_{n}: n \in \omega\right\rangle$ that consists of positive reals and converges to 0 a null sequence.

Proposition 1.1. Let $x$ be a point of $\mathbb{I}_{u}$ for which $A_{x} \cap B_{x}=\varnothing$. Then the following are equivalent:

(1) The point $x$ is a cut point of $\mathbb{I}_{u}$. 
(2) The sets of the form $\operatorname{Ex} \bigcup_{n \in U}(a(n), b(n))$, where $U \in u, a \in A_{x}$, and $b \in B_{x}$, form a local base at $x$.

(3) For every null sequence $\left\langle r_{n}: n \in \omega\right\rangle$ there are $a \in A_{x}$ and $b \in B_{x}$ such that the set $U=\left\{n: b(n)-a(n)<r_{n}\right\}$ belongs to $u$.

Proofs can be found in Hart [4] and Zhu [10].

Before we continue we would like to insert a remark that may take away the confusion that tends to be caused by condition (2) in the previous proposition.

Remark 1.2. Consider the following two conditions that one may impose on a point $x$ of $\mathbb{I}_{u}$ for which $A_{x} \cap B_{x}=\varnothing$ :

( $\alpha$ ) If $F$ is closed in $\mathbb{M}$ and $x \notin \operatorname{cl} F$ then there are $a \in A_{x}, b \in B_{x}$, and $U \in U$ such that $F(a, b, U)=\bigcup_{n \in U}[a(n), b(n)]$ is disjoint from $F$.

( $\beta$ ) If $F$ is closed in $\mathbb{M}$ and $x \in \operatorname{cl} F$ then there are $a \in A_{x}, b \in B_{x}$, and $U \in u$ such that $F(a, b, U)$ is contained in $F$.

Note that condition ( $\alpha$ ) is equivalent to condition (2) in Proposition 1.1 and hence characterizes nontrivial cut points. Condition $(\beta)$ on the other hand is stronger than $(\alpha)$ but not equivalent to it; in $[9,10]$ Zhu calls points that satisfy ( $\beta$ ) simple cut points.

In Section 2 we shall need the following variation of condition (3):

Lemma 1.3. A point $x$ of $\mathbb{I}_{u}$ with $A_{x} \cap B_{x}=\varnothing$ is a cut point if and only if for every $f \in \omega^{\omega}$ with $f(n)>0$ for all $n$ there is $g \in \omega^{\omega}$ with $g(n)<f(n)$ for all $n$ and such that the closed set $\bigcup_{n}[g(n) / f(n),(g(n)+1) / f(n)]$ belongs to $x$.

Proof. The condition of the lemma clearly implies condition (3) of Proposition 1.1 .

For the other direction fix $f$ and pick $a \in A_{x}$ and $b \in B_{x}$ such that $b(n)-$ $a(n)<1 / f(n)$ for all $n$. Now choose $g$ such that $g(n) \leqslant a(n) \cdot f(n)<$ $g(n)+1$ for all $n$. Then either $g$ or $g+1$ will do.

We now investigate how close to trivial a nontrivial cut point can be.

A consequence of condition (3) in Proposition 1.1 is that a nontrivial cut point is not in the closure of any closed discrete subset of $\mathbb{M}$, i.e., every nontrivial cut point is a far point. (A far point is a point that is not near.)

Indeed, if $D$ is closed and discrete in $\mathbb{M}$ then $D_{n}=D \cap \mathbb{I}_{n}$ is finite for every $n$. For each $n$ let $r_{n}$ be the minimum distance between two adjacent points of $D_{n}\left(r_{n}=1\right.$ if $\left.D_{n}=\varnothing\right)$. Now let $x$ be a nontrivial cut point of $\mathbb{I}_{u}$ and take $a \in A_{x}$ and $b \in B_{x}$ such that $b(n)-a(n)<r_{n}$ for all $n$. Then $\bigcup_{n}(a(n), b(n))$ picks at most one point from each $D_{n}$; but this gives us a set that, by definition, $x$ has to avoid.

On the other hand, under $\mathrm{CH}$ or weaker one can find nontrivial cut points that are in the closure of nowhere dense subsets of $\mathbb{M}$ (see Hart [4] or Zhu [9]; Theorem 1.5 provides another example).

To see what kind of sets can still support nontrivial cut points we consider scattered sets, as these are arguably the topologically smallest type of subsets of $\mathbb{R}$.

We assume the reader is familiar with the notion of a scattered space. We use $X^{(\alpha)}$ to denote the $\alpha$-th derived set of $X$. If $X$ is compact then the last $\alpha$ 
for which $X^{(\alpha)} \neq \varnothing$ is the scattered height of $X$, denoted ht $(X)$. Furthermore, if $x \in X$ then the last $\alpha$ for which $x \in X^{(\alpha)}$ is called the scattered rank of $x$.

Consider now a scattered closed subset $D$ of $\mathbb{M}$. We define its scattered height as $\mathrm{ht}(D)=\sup _{n} \mathrm{ht}\left(D \cap \mathbb{I}_{n}\right)$. We have seen that $D$ cannot support a nontrivial cut point if $D$ is discrete or, equivalently, if its scattered height is 0 . The same can be said if its scattered height is finite; this follows from the following proposition.

Proposition 1.4. If $x$ is a far point and $D$ is a closed subset of $M$ of finite scattered height then $x \notin \mathrm{cl} D$.

Proof. The argument is by induction on height: If the height of $D$ is $k$ then $D^{(k)}$ is closed and discrete, hence $x$ is not in its closure and there is a neighborhood, $X$, of it that is disjoint from $D^{(k)}$. The scattered height of $X \cap D$ is then at most $k-1$, so by our inductive assumption $x$ is not in the closure of $X \cap D$. It follows that $x$ is not in the closure of $D$.

The next result shows that this is best possible; under $\mathrm{CH}$ there is a closed set of scattered height $\omega$ that supports a nontrivial cut point for every $\mathbb{I}_{u}$. This theorem also solves, negatively, Question 13.2 from Hart [4], which asks for an $\mathbb{I}_{u}$ without nontrivial cut points.

Theorem 1.5 $(\mathrm{CH})$. Take, for each $n$, a copy $K_{n}$ of the ordinal space $\omega^{n}+1$ in $\mathbb{I}_{n}$. Then for every $u \in \omega^{*}$ there is a nontrivial cut point of $\mathbb{I}_{u}$ that is in the closure of $\bigcup_{n} K_{n}$.

Proof. We shall construct two sequences $\left\langle a_{\alpha}: \alpha \in \omega_{1}\right\rangle$ and $\left\langle b_{\alpha}: \alpha \in \omega_{1}\right\rangle$ in $\mathbb{I}^{\omega}$ such that:

(1) If $\alpha \in \beta \in \omega_{1}$ then $a_{\alpha}<^{*} a_{\beta}<^{*} b_{\beta}<^{*} b_{\alpha}$.

(2) For every null sequence $\left\langle r_{n}\right\rangle_{n}$ there is $\alpha \in \omega_{1}$ such that $b_{\alpha}(n)-a_{\alpha}(n)<$ $r_{n}$ for all but finitely many $n$.

(3) For every $x \in \mathbb{I}^{\omega}$ there is $\alpha \in \omega_{1}$ such that $x(n) \notin\left[a_{\alpha}(n)\right.$, $\left.b_{\alpha}(n)\right]$ for all but finitely many $n$.

To avoid cumbersome notation we shall use $I(\alpha, n)$ to denote both $\left[a_{\alpha}(n)\right.$, $\left.b_{\alpha}(n)\right]$ and $\{n\} \times\left[a_{\alpha}(n), b_{\alpha}(n)\right]$; the context should always dictate which meaning we use.

Consider now the closed set

$$
C=\mathbb{M}^{*} \cap \bigcap_{\alpha \in \omega_{1}} \mathrm{cl} \bigcup_{n \in \omega} I(\alpha, n) .
$$

Condition (1) implies that $C$ meets every $\mathbb{I}_{u}$, condition (2) implies that $C \cap \mathbb{I}_{u}$ consists of exactly one point for every $u$, and condition (3) implies that every such point is a nontrivial cut point (see Proposition 1.1). To make sure that $C$ is a subset of the closure of $\bigcup_{n} K_{n}$ we are forced, by Proposition 1.4, to add the following condition to our list.

(4) For every $\alpha$ we have $\lim _{n \rightarrow \infty}$ ht $\left(I(\alpha, n) \cap K_{n}\right)=\infty$.

Now let $\left\{x_{\alpha}: \alpha \in \omega_{1}\right\}$ list $\mathbb{I}^{\omega}$ and let $\left\{c_{\alpha}: \alpha \in \omega_{1}\right\}$ list the set of all null sequences. During the construction we make sure that for every $\alpha$ the set

$$
\left\{n: x_{\alpha}(n) \in I(\alpha, n) \text { and } b_{\alpha}(n)-a_{\alpha}(n) \geqslant c_{\alpha}(n)\right\}
$$

is finite. 
We start by putting $a_{-1}(n)=0$ and $b_{-1}(n)=1$ for all $n$.

Now let $\alpha \in \omega_{1}$ and assume everything has been taken care of below $\alpha$. If $\alpha$ is a successor or 0 put $a^{\alpha}=a_{\alpha-1}$ and $b^{\alpha}=b_{\alpha-1}$. If $\alpha$ is a limit first choose an increasing cofinal sequence $\left\langle\gamma_{i}: i \in \omega\right\rangle$ in $\alpha$. Then choose an increasing sequence $\left\langle n_{i}: i \in \omega\right\rangle$ in $\omega$ such that for every $i:$ if $n \geqslant n_{i}$ then $\operatorname{ht}\left(I\left(\gamma_{i}, n\right) \cap K_{n}\right) \geqslant i$ and if $j<i$ then $a_{\gamma_{j}}(n)<a_{\gamma_{i}}(n)$ and $b_{\gamma_{j}}(n)>b_{\gamma_{i}}(n)$.

Now define $a^{\alpha}$ and $b^{\alpha}$ by: if $n<n_{0}$ then $a^{\alpha}(n)=0$ and $b^{\alpha}(n)=1$, and: if $n_{i} \leqslant n<n_{i+1}$ then $a^{\alpha}(n)=a_{\gamma_{i}}(n)$ and $b^{\alpha}(n)=b_{\gamma_{i}}(n)$. In either case $\lim _{n \rightarrow \infty} \mathrm{ht}\left(\left[a^{\alpha}(n), b^{\alpha}(n)\right] \cap K_{n}\right)=\infty$.

Choose, for every $n$ a point $y_{n}$ in $\left[a^{\alpha}(n), b^{\alpha}(n)\right] \cap K_{n}$ of maximum scattered rank, say $k_{n}$, and an interval $J_{n}$ around $y_{n}$ of length at most $c_{\alpha}(n)$ and contained in $\left[a^{\alpha}(n), b^{\alpha}(n)\right]$. Now pick, if $k_{n} \geqslant 1$, a point $z_{n}$ in $J_{n}$ of rank $k_{n}-1$ but not equal to $x_{\alpha}(n)$. Finally then choose $a_{\alpha}$ and $b_{\alpha}$ in such a way that $I(\alpha, n)$ has $z_{n}$ in its interior but does not contain $x_{\alpha}(n)$ whenever $k_{n} \geqslant 1$, in the finitely many cases when $k_{n}=0$ the choice of $a_{\alpha}(n)$ and $b_{\alpha}(n)$ is immaterial. This concludes the proof.

Remark 1.6. We note that a suitable modification of the arguments of Baldwin and Smith from [1] can be used to show that under Martin's Axiom for Countable posets there is some $u \in \omega^{*}$ for which $\mathbb{I}_{u}$ has a nontrivial cut point that is in the closure of a scattered set of height $\omega$.

Our stronger assumption of $\mathrm{CH}$ yields a stronger conclusion: every $\mathbb{I}_{u}$ has such a nontrivial cut point.

Let us summarize what kind of nontrivial cut points we can have.

To begin, every nontrivial cut point is a far point and hence not in the closure of a set of finite scattered height. On the other hand, we just constructed a nontrivial cut point in the closure of a set of scattered height $\omega$.

The point constructed by Baldwin and Smith is remote, which means that it is not in the closure of any closed nowhere dense subset of $\mathbb{M}$. Finally, the point constructed by $\mathrm{Zhu}$ is not remote but still quite far-it has a base of perfect sets. We remark that a cut point is remote iff it is simple, i.e., satisfies condition ( $\beta$ ) of Remark 1.2.

In [10] Zhu showed that in Laver's model there are no remote cut points; in the next section we show that in this model there are no nontrivial cut points at all, by showing that no far point is a cut point.

\section{A MODEL WITHOUT NONTRIVIAL CUT POINTS}

In this section we prove that there are no nontrivial cut points in Laver's model for the Borel conjecture. We need to describe Laver's poset of course.

To begin, a Laver tree is a subtree $T$ of ${ }^{<\omega} \omega$ of the following form: There is a node $s_{T}$ of $T$ such that for every $t \in T$ either $s_{T} \subseteq t$ or $t \subseteq s_{T}$; we call $s_{T}$ the root node of $T$. Furthermore, if $t \in T$ extends $s_{T}$ then the set of $i \in \omega$ for which $t^{\frown} i \in T$ is infinite. The set of branches through $T$ is denoted by $[T]$.

The Laver poset $\mathbb{L}$ is the set of Laver trees, ordered by inclusion. If $G$ is a generic filter on $\mathbb{L}$ then it determines a new real, a Laver real, as follows: 
In $V[G]$ the intersection $\bigcap[T]: T \in G\}$ consists of one point $f$; this point $f$ is the Laver real and it determines $G$ because $G=\{T: f \in[T]\}$. The function $f$ goes much faster to infinity than any function from $V$ and this is what we shall use to destroy nontrivial cut points.

A crucial property of Laver forcing is the following.

Lemma 2.1. If $T \in \mathbb{L}, F$ is a finite set in $V$ and $T \Vdash \dot{x} \in F$ then there are $S \leqslant T$ with the same root as $T$ and $a \in F$ such that $S \Vdash \dot{x}=a$.

Laver's model is obtained from a model of $\mathrm{CH}$, using a countable-support iterated forcing construction of length $\omega_{2}$ where each time one forces with the poset $\mathbb{L}$.

Theorem 2.2. In Laver's model there are no nontrivial cut points.

Proof. Let $\left\langle\mathbb{P}_{\alpha}: \alpha \leqslant \omega_{2}\right\rangle$ be a countable support iteration, where at every stage $\mathbb{P}_{\alpha+1}=\mathbb{P}_{\alpha} * \dot{\mathbb{L}}$. We let $\mathbb{P}=\mathbb{P}_{\omega_{2}}$ and we let $G$ be a generic filter on $\mathbb{P}$.

Finally we assume that, in $V[G], x$ is a far point of $\mathbb{M}$; we must show that $x$ is not a cut point of the $\mathbb{I}_{u}$ that contains it.

An easy reflection argument will produce an ordinal $\alpha<\omega_{2}$ such that, in the model $V[G\lceil\alpha]$, the point $x\lceil\alpha$ is a far point of $\mathbb{M}$. We show that, in $V[G]$, there is no nontrivial cut point extending the filter $x \mid \alpha$ (in particular, $x$ is not a cut point).

For this we consider the Laver real $f$, added at the next stage. It induces partitions of the intervals $\mathbb{I}_{n}$ :

$$
\mathbb{I}_{n}=\bigcup\{[i / f(n),(i+1) / f(n)]: i<f(n)\} .
$$

In what follows we shall denote the interval $[i / f(n),(i+1) / f(n)]$ by $I(n, i)$.

Our task will be complete once we show that for every $g: \omega \rightarrow \omega$ from $V[G]$ satisfying $(\forall n \in \omega)(g(n)<f(n))$ there is an $X \in x\lceil\alpha$ disjoint from the set $\bigcup_{n} I(n, g(n))$.

The intuition behind this is that $f$ grows so fast that the intervals $I(n, g(n))$ become very thin, as thin as points. As $x$ is a far point, we must then be able to avoid those intervals.

For convenience we drop (as we may) all references to $\alpha$ and simply assume that we have a far point $x$ in $V$ and show that it has the property mentioned in the previous paragraph.

So assume that $g$, from $V[G]$, is a function below the first Laver real $f$. A straightforward application of Lemma 6 from Laver [6] gives us, in $V[f]$, a function $F$ such that $F(n)$ is an $n$-element subset of $f(n)$ and $g(n) \in F(n)$ for all $n$.

In $V$ this gives us a $T \in \mathbb{L}$ that forces all this:

$$
T \Vdash_{\mathbb{L}}(\forall n \in \omega)(\dot{F}(n) \subseteq \dot{f}(n) \wedge|\dot{F}(n)|=n),
$$

and there is a condition $p \in \mathbb{P}$, with first coordinate $T$, such that

$$
p \Vdash_{\mathbb{P}}(\forall n \in \omega)(\dot{g}(n) \in \dot{F}(n)) .
$$


We will be done once we show that the set of $S \in \mathbb{L}$ for which there is $X \in X$ such that

$$
S \Vdash_{\mathbb{L}} X \cap \bigcup\{I(n, i): i \in \dot{F}(n), n \in \omega\}=\varnothing
$$

is dense below $T$. It suffices to find such an $S$ below $T$ (the same argument works below any other element below $T$ ).

We begin by observing that if $t \in T$ and $t^{\frown} i \in T$ then

$$
T \uparrow\left(t^{\frown} i\right) \Vdash \dot{f}(|t|)=i
$$

and hence

$$
T \uparrow\left(t^{\frown} i\right) \Vdash \dot{F}(|t|) \subseteq i .
$$

Therefore we may, using Lemma 2.1, assume that we have a partial function $H: T \times \omega \rightarrow[\omega]^{<\omega}$ such that

$$
T \uparrow\left(t^{\frown} i\right) \Vdash \dot{F}(|t|)=H(t, i) .
$$

We enumerate $H(t, i)$ in increasing order as $\{h(t, i, j): j<|t|\}$ and we denote the interval $[h(t, i, j) / i,(h(t, i, j)+1) / i]$ by $I(t, i, j)$. It should be clear that all we need is a Laver tree $S$ below $T$ and an element $X$ of $x$ such that

$$
X \cap(\{n\} \times I(t, i, j))=\varnothing
$$

whenever $n, t, i$, and $j$ are such that $t^{\frown} i \in S,|t|=n$, and $j<n$. This then is the goal of the rest of the proof.

Let $n_{0}$ be the length of the root node of $T$ and denote the root node by $t_{n_{0}}$. We shall thin out $T$ by induction on $n \geqslant n_{0}$. At each step, when we have a tree $T_{n-1}$, we choose a node $t_{n}$ of $T_{n-1}$ and we make sure that the nodes $t_{0}, \ldots, t_{n}$ will be in the tree $T_{n}$. Furthermore the choice will be made in such a way that in the end the tree $T_{\infty}=\left\{t_{n}: n \in \omega\right\}$ is a Laver tree with root $t_{n_{0}}$ (for $n<n_{0}$ we let $t_{n}=t_{n_{0}}\lceil n$ ). This amounts to what is generally called a 'standard fusion argument'; the $t_{n}$ can be chosen in a very canonical way, described in detail in $[6$, p. 156].

At step $n_{0}$ we choose an infinite subset of $\left\{i: t_{n_{0}} \wedge i \in T\right\}$ such that the sequence of cubes $\prod_{j<n_{0}} I\left(t_{n_{0}}, i, j\right)$ converges to a point $r_{n_{0}}\left(t_{n_{0}}\right)$ of $\mathbb{I}^{n_{0}}$. After throwing away the other $i$ 's we end up with the tree $T_{n_{0}}$.

Now let $n>n_{0}$. Consider the tree $T_{n-1}$ and choose $t_{n}$ in $T_{n-1}$ as an immediate successor of some $t_{k}$ with $n_{0} \leqslant k<n$. Using the nodes $t_{k}$ we split $T_{n-1}$ into subtrees: if $n_{0} \leqslant k \leqslant n$ then $T_{n, k}$ is the union of all $T\lceil t$ where $t$ is an immediate successor of $t_{k}$, but none of the $t_{j}$ for $k<j \leqslant n$ (note that $t_{k}$ is the root node of $T_{n, k}$ ).

Fix $k$ between $n_{0}$ and $n$. For every $t \in \operatorname{Lev}\left(T_{n, k}, n\right)$ we choose an infinite subset of $\left\{i: t^{\frown} i \in T_{n, k}\right\}$ such that the cubes $\prod_{j<n} I(t, i, j)$ converge to a point $r_{n}(t)$ of $\mathbb{I}^{n}$ and we throw away the parts of $T_{n, k}$ above the other successors of $t$. Note that such a choice is possible because for every fixed $t$ the diameters of the cubes $\prod_{j<n} I(t, i, j)$ converge to 0 . We continue down to the level of $t_{k}$, all the time thinning out $T_{n, k}$ further to a tree $T_{n, k}^{\prime}$ with 
the property that for every $t$ with $\left|t_{k}\right| \leqslant|t|<n$ the sequence

$$
\left\langle r_{n}\left(t^{\frown} i\right): t^{\frown} i \in T_{n, k}^{\prime}\right\rangle
$$

converges to a point $r_{n}(t) \in \mathbb{I}^{n}$.

When this is done for every $k$ we piece the trees $T_{n, k}^{\prime}$ together to form the tree $T_{n}$.

In the end we get the tree $T_{\infty}=\bigcap_{n} T_{n}=\left\{t_{n}: n \in \omega\right\}$. It gives us, for $n \geqslant n_{0}$, the following picture in $\mathbb{I}^{n}$ (it may be better to think of this as taking place in $\left.\mathbb{I}_{n}^{n}\right)$ : for every $k \in\left[n_{0}, n\right]$ a point $r_{n}\left(t_{k}\right)$ with a sequence $\left\langle r_{n}\left(t^{\frown} i\right)\right.$ : $\left.t^{\curvearrowleft} i \in T_{\infty}\right\rangle$ converging to it. Each of the terms of this sequence has a sequence converging to it and so on until we reach the points above $t_{k}$ that are on level $n$, then we get sequences of cubes converging to the corresponding $r_{n}(t)$.

In $\mathbb{I}_{n}$ we get the finite set of coordinates of the points $r_{n}\left(t_{k}\right)$ :

$$
F_{n}=\left\{\left\langle n, r_{n}\left(t_{k}, j\right)\right\rangle: n_{0} \leqslant k \leqslant n, j<n\right\},
$$

together with sequences converging to them:

$$
G_{n}=\left\{\left\langle n, r_{n}\left(t_{k} \frown i, j\right)\right\rangle: n_{0} \leqslant k \leqslant n, t_{k} \frown i \in T_{\infty}, j<n\right\} .
$$

Because $x$ is a far point of $\mathbb{M}$ we may find an element $X$ of $x$ that is disjoint from $\bigcup_{n}\left(F_{n} \cup G_{n}\right)$ (each $F_{n} \cup G_{n}$ has scattered height 1). Our intuition did not let us down, we will manage to confine $g$ near the set $\bigcup_{n}\left(F_{n} \cup G_{n}\right)$.

Now we are ready for the final recursive trim.

The points $\left\langle n_{0}, r_{n_{0}}\left(t_{n_{0}}, j\right)\right\rangle$ are not in $X$, so for all but finitely many $i$ with $t_{n_{0}} \frown i \in T_{\infty}$ every interval $\left\{n_{0}\right\} \times I\left(t_{n_{0}}, i, j\right)$ is disjoint from $X$. Discard those finitely many $i$.

Now let $t_{m}$ be one of the surviving direct successors of $t_{n_{0}}$. The property that allows the trimming to continue is that $\left\langle n, r_{n}\left(t_{m}, j\right)\right\rangle \notin X$ for all $n \geqslant\left|t_{m}\right|$ (for $n \geqslant m$ use the fact that $\left\langle n, r_{n}\left(t_{m}, j\right)\right\rangle \in F_{n}$ and for $n<m$ use the fact that $\left\langle n, r_{n}\left(t_{m}, j\right)\right\rangle \in G_{n}$; it's in the sequence converging to $\left.\left\langle n, r_{n}\left(t_{n_{0}}, j\right)\right\rangle\right)$.

For every $n$ from $\left|t_{m}\right|+1$ through $m$ we have to discard those finitely many successors $t$ of $t_{m}$ for which some $\left\langle n, r_{n}(t, j)\right\rangle$ is in $X$ and any of the finitely many more $t=t_{m} \wedge i$ for which $\left\{\left|t_{m}\right|\right\} \times I\left(t_{m}, i, j\right)$ meets $X$. For $n>m$ we have $\left\langle n, r_{n}\left(t_{m}, j\right)\right\rangle \in F_{n}$, hence for all direct successors $t$ of $t_{m}$ we have $\left\langle n, r_{n}(t, j)\right\rangle \in G_{n}$ and so no more trimming of successors of $t_{m}$ is required.

The same strategy applies to nodes higher up in $T_{\infty}$ : whenever a node $t_{k}$ has survived it will lose finitely many direct successors. These successors must be discarded because of possible nonempty intersections with $X$ in $\mathbb{I}_{n}$ for $\left|t_{k}\right| \leqslant n \leqslant k$. In the end we get our tree $S$ satisfying $(*)$.

To end this paper we show how Theorem 2.2 may be applied in the theory of far points of $\mathbb{H}=[0, \infty)$. The theorem implies that in Laver's model a point of $\mathbb{H}^{*}$ is a near point if and only if it is a sub cut point and hence that the set of near points is topologically invariant in $\mathbb{H}^{*}$.

This is a partial answer to a question of van Douwen from [3] whether the set of remote points of $\mathbb{H}$ is topologically invariant. Under $\mathrm{CH}$ it is not: in [8] $Y u$ showed that if $u$ is a $P$-point then any two cut points of $\mathbb{I}_{u}$ can be mapped 
to each other by an autohomeomorphism of $\mathbb{M}^{*}$ that leaves every $\mathbb{I}_{v}$ invariant; so, for example, a remote point can become a near point. This can then be modified to produce an autohomeomorphism of $\mathbb{H}^{*}$ with the same effect.

This suggests the obvious question whether the set of remote points of $\mathbb{H}^{*}$ is topologically invariant in Laver's model.

\section{REFERENCES}

1. S. Baldwin and M. Smith, On a possible property of far points of $\beta[0, \infty)$, Topology Proc. 11 (1986), 239-245.

2. E. K. van Douwen, Subcontinua and nonhomogeneity of $\beta \mathbb{R}^{+}-\mathbb{R}^{+}$, Notices Amer. Math. Soc. 24 (1977), 77T-G114, A-559.

3. __ Remote points, Dissertationes Math. 188 (1981), 1-45.

4. K. P. Hart, The Čech-Stone compactification of the real line, Recent Progress in General Topology (M. Hušek and J. van Mill, eds.), Chapter 9, North-Holland, Amsterdam, 1992, pp. 317-352.

5. K. P. Hart and J. van Mill, Open problems on $\beta \omega$, Open Problems in Topology (J. van Mill and G. M. Reed, eds.), Chapter 7, North-Holland, Amsterdam, 1990, pp. 97-125.

6. R. Laver, On the consistency of Borel's Conjecture, Acta Math. 137 (1976), 151-169.

7. J. van Mill and C. F. Mills, A topological property enjoyed by near points but not by large points, Topology Appl. 11 (1980), 199-209.

8. J. Y.-C. Yu, Autohomeomorphism in the Stone-Čech remainder of the reals, preprint, 1991.

9. J.-P. Zhu, On indecomposable subcontinua of $\beta[0, \infty)-[0, \infty)$, Topology Appl. 45 (1992), 261-274; Proceedings of the Tsukuba Topology Symposium, Tsukuba University, 1990.

10.

Department of Mathematics, York University, 4700 Keele Street, North York, Ontario, Canada M3J 1P3

E-mail address: dowa@nexus. yorku.ca

Faculty of Technical Mathematics and Informatics, TU Delft, Postbus 5031, 2600 GA DeLFT, THE NetherLANDS

E-mail address: wiawkph@dutrun2.tudelft.nl 